\title{
Presencia de metalo-ß-lactamasas en Pseudomonas aeruginosa resistente a imipenem
}

\author{
Alfonso Pérez I ${ }^{1 a}$, Patricia García $C^{2}$, Helena Poggi M ${ }^{3 b}$, \\ Stephanie Braun J ${ }^{4}$, Claudia Castillo V3c, Juan Carlos Román 3c, \\ Marcela Lagos ${ }^{2}$, Eliana Romeo $0^{3 \mathrm{C}}$, Lorena Porte $\mathrm{T}^{4}$, \\ Jaime Labarca $L^{5}$, G erardo G onzález $\mathbf{R}^{6 \mathbf{d}}$. \\ Presence of metallo ß-lactamases \\ in imipenem-resistant \\ Pseudomonas aeruginosa
}

Background: Metallo- $\beta$-lactamases (MBL) confer high resistance to carbapenems in Pseudomonas aeruginosa (Psae). They are encoded in mobile elements of different genes (VIM, IMP, SMP, GIM), along with other resistance genes. Aim: To detect the presence of MBL in imipenem resistant Psae strains. Material and methods: Fifty-nine imipenem resistant Psae strains isolated from January 2004 to August 2005 in a University Clinical Hospital, were included. The presence of MBL was studied by Etest (phenotypic) and genotypic polymerase chain reaction (PCR) methods. To rule out a nosocomial outbreak, MBL positive strains, were studied by pulse field gel electrophoresis. Results: The presente of MBL was detected in eleven strains. All were type VIM and were not clonally related. There was no concordance between phenotypic and genotypic MBL detecting methods. All the strains were also multiresistant. Conclusions: The presence of MBL was detected in 19\% of imipenem resistant Psae strains (Rev Méd Chile 2008; 136: 423-32).

(Key w ords: Beta-Lactamases; Imipenem; Pseudomonas aeruginosa)

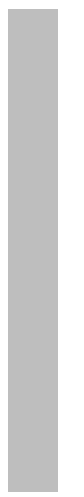

\footnotetext{
Recibido el 25 de junio, 2007. Aceptado el 12 de octubre, 2007.

Fuente financiamiento: Proyectos de Investigación Unidad Docente Asociada 2005, PUC

${ }^{1}$ Escuela de Medicina. Pontificia Universidad Católica de Chile. Santiago de Chile. ${ }^{2}$ Departamento de Laboratorios Clínicos. Facultad de Medicina. Pontificia Universidad Católica de Chile. Santiago de Chile. ${ }^{3}$ Laboratorio de Microbiología y Laboratorio de Biología Molecular. Servicio de Laboratorios Clínicos. Pontificia Universidad Católica de Chile. Santiago de Chile. ${ }^{4}$ Laboratorio de Microbiología. Hospital Militar. Santiago de Chile. ${ }^{5}$ Departamento de Medicina Facultad de Medicina. Pontificia Universidad Católica de Chile. Santiago de Chile. ${ }^{6}$ Departamento de Microbiología, Universidad de Concepción. Concepción, Chile.

${ }^{a}$ Licenciado en Medicina

bBioquímico

'Tecnólogo médico

${ }^{\mathrm{d}}$ Lic. Biología, PhD. Ciencias biológicas
}

Correspondencia a: Dra. Patricia García. Laboratorio de Microbiología, Centro Médico San Joaquín. Av. Vicuña Mackenna 4686, Macul, Santiago de Chile. Fono: 56-2-3548576. Fax: 56-2-354-8571. E mail: pgarcia@med.puc.cl 
$\mathrm{P}$ seudomonas aeruginosa es uno de los principales agentes aislados de infecciones intrahospitalarias, entre ellas, neumonía asociada a ventilación mecánica, infecciones urinarias, de piel y partes blandas. Según diversos estudios realizados en Latinoamérica bajo el Programa de Vigilancia de Susceptibilidad Antimicrobiana SENTRY ${ }^{1-4}$, COrresponde al 5ㅇaente más frecuentemente aislado en bacteriemias.

En los últimos años se ha observado un aumento de la prevalencia de cepas de Pseudomonas aeruginosa resistentes a carbapenémicos, las que a su vez presentan resistencia múltiple a otros antibióticos 5 .

Datos chilenos provenientes de diversos estudios ${ }^{1,4,6,7}$ describen tasas de resistencia a carbapenémicos que varían entre 3\% y 25\%. En un estudio reciente realizado en Santiago, en 2004, en los hospitales Militar y Clínico de la Universidad Católica de Chile, se encontró 17\% de resistencia a imipenem en 338 aislados clínicos de Pseudomonas aeruginosa 8 .

En la Red Nacional de Vigilancia de Resistencia a los Antimicrobianos, entre los años 2004 y 2005, en 11 hospitales de Chile, se vigilaron infecciones invasoras e infecciones respiratorias bajas, correspondiendo $\mathrm{P}$ aeruginosa a $15 \%$ de los aislados intrahospitalarios. En estos aislados, la susceptibilidad a imipenem alcanzó 80\% (Red de Resistencia, II Curso de Resistencia Bacteriana, 2005, Santa Cruz, Chile, datos no publicados).

La resistencia de Pseudomonas aeruginosa a carbapenémicos puede ser explicada por diversos mecanismos:

1. Producción de metalo ß-lactamasas (MBLs). 2. Excreción del antibiótico mediante sobreexpresión de bombas de eflujo (MexAB-OprM) comprometiéndose en este caso, la sensibilidad a meropenem más que imipenem y la de otros antibióticos no carbapenémicos (fluoroquinolonas, penicilinas, cefalosporinas). 3. Alteraciones de la porina OprD que confiere impermeabilidad a carbapenémicos, generando resistencia a imipenem y susceptibilidad disminuida a meropenem.

Las MBLs tienen un amplio espectro hidrolítico, inactivando a todos los beta-lactámicos, incluso, los carbapenémicos y exceptuando el aztreonam. Son muy eficientes sobre imipenem, aumentando las concentraciones inhibitorias mínimas (CIM) a más de $32 \mathrm{ug} / \mathrm{ml}$. Sin embargo, su acción hidrolítica es dependiente de zinc $\left(\mathrm{Zn}^{+2}\right)$, por lo que quelantes como el ácido etilendiaminotetraacético (EDTA) las inhiben, disminuyendo las $\mathrm{CIM}^{9-11}$.

Se han descrito cuatro tipos de MBLs clínicamente relevantes: IMP, SMP, GIM y VIM. La mayoría de ellas han sido reportadas en Europa y Asia, encontrándose también algunas subclases de VIM en Estados Unidos de Norteamérica ${ }^{11-13}$. En Latinoamérica no habían sido descritas hasta 2003, fecha en que se identificó el gen bla $\mathrm{VIM}-2$ codificante para la MBL VIM-2, en 1 cepa de Pseudomonas flourescens de Chile y 3 cepas de Pseudomonas aeruginosa de Venezuela resistentes a imipenem ${ }^{14}$.

La resistencia de $\mathrm{P}$ aeruginosa a carbapenémicos por este mecanismo ha ido en aumento, llegando a ser responsable de hasta $40 \%$, con una amplia diseminación a nivel mundial. Desde el punto de vista clínico esto es importante, ya que muchas de estas son multirresistentes con limitadas alternativas terapéuticas (como polimixina $\mathrm{B}$ y colistin) y desde el punto de vista epidemiológico pueden manifestarse como brotes nosocomiales ${ }^{11,15,16}$.

Dado que no se tenía información sobre MBLs en Chile desde su descripción en 2003, los objetivos de este estudio fueron detectar la presencia y la frecuencia de MBLs en Pseudomonas aeruginosa resistentes a imipenem en un hospital universitario, así como también determinar si los perfiles de resistencia en cepas portadoras de MBLs difieren del que presentan las cepas resistentes por otros mecanismos (no MBL).

\section{MATERIAL Y MÉTODO}

Selección de cepas. Entre enero de 2004 y agosto de 2005 se aislaron en forma sucesiva 834 cepas de Pseudomonas aeruginosa, provenientes de muestras clínicas procesadas en el Laboratorio de Microbiología del Hospital Clínico de la Universidad Católica de Chile en Santiago. Se encontró que de las 834 cepas de P aeruginosa, 128 (15,3\%) fueron resistentes a imipenem por método de dilución en agar. En todas las cepas se confirmó la resistencia por un método alternativo $\left(\right.$ Etest $\left.^{\circledR}\right)$. Las cepas fueron almacenadas a $-20^{\circ} \mathrm{C}$ hasta su análisis. De las 128, sólo 65 estuvieron disponibles para estudio. De éstas, se analizaron 59 cepas, por ser consideradas eventos infecciosos independien- 
tes (muestras provenientes de distinto sitio de aislamiento, con un intervalo mayor a una semana) correspondientes a 51 pacientes.

Detección de MBLs. a) Método genotípico: reacción en cadena de la polimerasa (RPC). En las 59 cepas seleccionadas se estudió la presencia de MBLs por RPC con dos parejas de partidores comunes para los distintos tipos del gen bla $_{\mathrm{VIM}}$ (Pareja A: VIM S: 5'-CCGATGGTGTTTGGTCGCAT- 3' y VIM AS: 5'GAATGCGCAGCACCAGGAT-3', amplificando un fragmento de $391 \mathrm{pb}$; Pareja B: VIM-F: 5'- AAAGTTATGCCGCACTCACC-3' y VIM-R 5'-TGCAACTTCATGTTATGCCG-3', fragmento amplificado de $864 \mathrm{pb)}$ y una pareja de partidores, común para los distintos tipos del gen bla IMP $_{\text {(IMP S: AAAGA- }}$ TACTGAAAAGTTAGT e IMP AS: TCYCCAAYTTCACTRTGACT, tamaño del fragmento amplificado: $446 \mathrm{pb})^{14,17}$. Se usaron como controles positivos las cepas Pseudomonas fluorescens 43-14926 y Serratia marcescens AK9373 portadoras de VIM e IMP, respectivamente ${ }^{14,18}$.

b) Método fenotípico: Se buscó la presencia de MBLs por Etest MBL de AB-Biodisk ${ }^{\circledR}$ (test de sinergia imipenem-EDTA). Se consideró el test positivo cuando la relación entre CIM de imipenem/imipenem + EDTA fue mayor o igual a 8 (según recomendación del fabricante).

Análisis de clonalidad. En las cepas con resultados positivos para MBLs se evaluó clonalidad por electroforesis en gel de campo pulsado según lo descrito por Maslow y Lutsky ${ }^{19}$ interpretada según los criterios de Tenover et $\mathrm{al}^{20}$. Además, fueron estudiadas por RAPD (sigla en inglés para Randomly amplified polymorphic DNA) con el partidor ERIC 2 (5': AAGTAAGTGACTGGGGTGAGCG) ${ }^{21}$.

Resistencia a antibióticos. Se evaluó la resistencia por el método de dilución en agar ${ }^{22}$ para 5 antibióticos: una cefalosporina con actividad antipseudomónica (ceftazidima), una fluoroquinolona (ciprofloxacino), un aminoglicósido (amikacina), un betalactámico asociado a un inhibidor de betalactamasas (cefoperazona/sulbactam) y otro carbapenémico (meropenem).

Se utilizaron los puntos de corte descritos por CLSI $^{23}$ excepto para cefoperazona/sulbactam, en el que se usó el punto de corte descrito por Jones et $\mathrm{al}^{24}$.
Se consideraron multirresistentes a aquellas cepas que además de presentar resistencia a imipenem, mostraron susceptibilidad disminuida a otro de los antimicrobianos probados 25 .

Se estudió la susceptibilidad a colistin por 2 métodos alternativos: difusión en agar (discos de 10 ug; BBL) y Etest AB-Biodisk ${ }^{\circledR}$. Se utilizaron los puntos de corte recomendados por el fabricante: sensible $\leq 4 \mathrm{ug} / \mathrm{ml}$, resistente $\geq 8 \mathrm{ug} / \mathrm{ml}^{26}$.

Estadística. El análisis estadístico se realizó con el software Medcalc versión 4.16e. La comparación de concentraciones inhibitorias mínimas (CIMs) a imipenem y la relación imipenem/ imipenem -

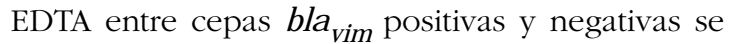
realizó por el test de Wilcoxon para muestras independientes. En estos grupos, también se analizó la susceptibilidad a antibióticos por el Test Chi-cuadrado. La multirresistencia fue evaluada por T-test. Se consideró estadísticamente significativo un valor $\mathrm{p}<0,05$.

\section{Resultados}

De las 59 cepas estudiadas, 47 (80\%) correspondían a pacientes hospitalizados y $12(20 \%)$ a pacientes ambulatorios, de estos últimos, ninguno tenía el antecedente de hospitalizaciones previas en nuestro hospital. El promedio de edad fue de 53 años (rango: 16 días a 95 años) y 40 de los pacientes $(67,8 \%)$ eran de sexo masculino. El origen clínico de las cepas fue en su mayoría muestras respiratorias: lavado bronquioalveolar: 7 , aspirado endotraqueal: 11 , expectoración: 17 , hemocultivos: 3, cultivo de punta de catéter: 1, urocultivos: 11 y otras: 9 .

\section{Detección de MBLs}

Método genotípico: RPC. Por este método se encontraron 11 cepas portadoras del bla $_{\mathrm{VIM}}$ $(18,6 \%)$ (Figura 1). Ambas parejas de partidores (VIM S-AS y VIM F-R) fueron igualmente efectivas en detectar el gen bla $_{\mathrm{VIM}}$, en las mismas portadoras. No se encontraron cepas portadoras del gen bla $_{\text {IMP }}$ (Figura 2).

Las once cepas positivas para el gen bla $\mathrm{VIM}_{\mathrm{M}}$ fueron aisladas de 10 pacientes. Dos eran ambulatorios y las nueve restantes de pacientes hospitalizados en siete servicios diferentes. Dos cepas 


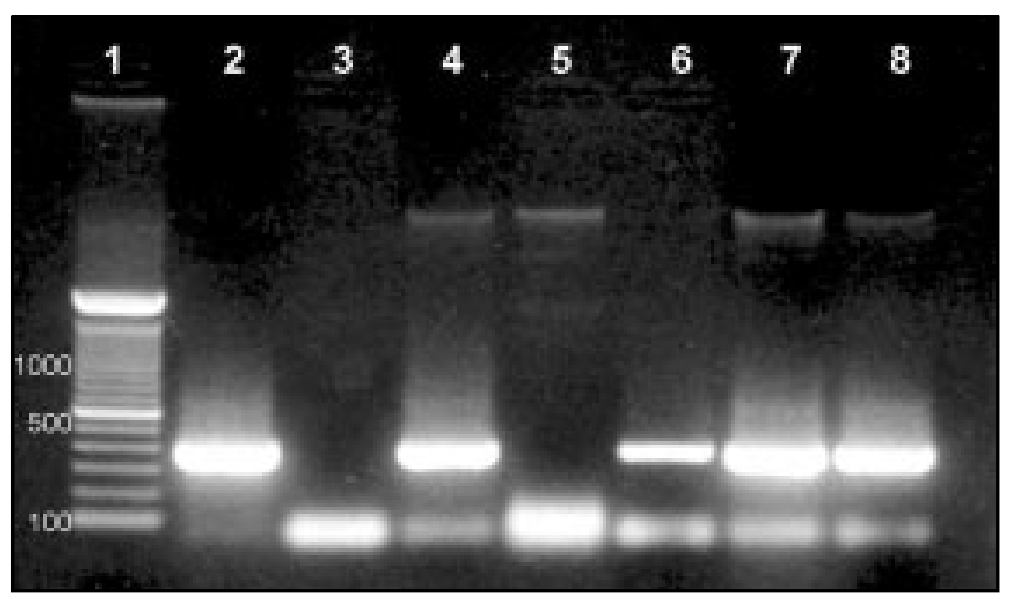

Figura 1. RPC bla ${ }_{\mathrm{VIM}}$ con pareja de partidores VIM S-AS. Carril 1: marcador de peso molecular, carril 2: control positivo (Pseudomonas flourescens 43-14926), carril 3: control negativo, carriles 4 al 8: cepas estudiadas. En los carriles 2, 4, 6, 7 y 8 se observa una banda correspondiente al fragmento de $391 \mathrm{pb}$ del gen bla VIM. $_{\text {. }}$

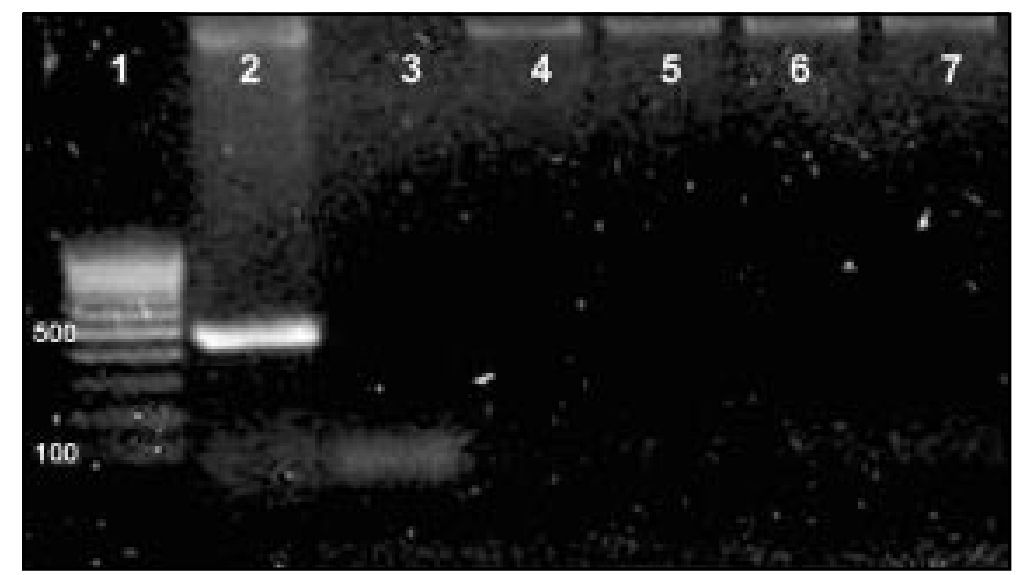

Figura 2: PCR bla ${ }_{\text {IMP }}$ con pareja de partidores IMP S e IMP AS. En carril 1: marcador de peso molecular, carril 2: control positivo (Serratia marcescens AK9373), carril 3: control negativo, carriles 4 al 7: cepas estudiadas. En el carril 2 se observa una banda correspondiente al fragmento de $446 \mathrm{pb}$ del gen bla IMP. $_{\text {. }}$.

correspondieron a un mismo paciente, pero fueron aisladas con un intervalo mayor a un año y en muestras de localizaciones distintas, constituyendo dos eventos independientes.

Método fenotípico: Etest. En 49 de las 59 cepas estudiadas $(83,1 \%)$, el test fenotípico resultó positivo (relación CIM imipenem/CIM imipenemEDTA mayor o igual a ocho veces). Todas las cepas positivas para el gen bla ${ }_{\mathrm{VIM}}$ por RPC lo fueron por Etest. Por otra parte, 38 de 48 (79,2\%) de las cepas negativas por RPC resultaron positivas por método fenotípico. De acuerdo con esto, la concordancia entre ambos métodos fue de 35,6\% (21 cepas) (Tabla 1).

No se encontró asociación entre la presencia del gen bla ${ }_{\mathrm{VIM}} \mathrm{y}$ las CIMs a imipenem, pero la relación CIM imipenem/ CIM imipenem-EDTA en

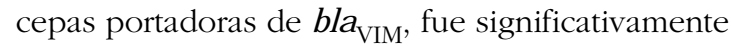
superior (Tabla 2).

2. Análisis de clonalidad y patrones de susceptibilidad en cepas portadoras del gen bla ${ }_{V I M}$ Las once cepas positivas para el gen bla $_{\text {VIM }}$ no mostraron relación clonal entre ellas, tanto en la 
Tabla 1. Comparación de método fenotípico (Etest) con método genotípico (RPC)

\begin{tabular}{|lccc|}
\hline $\begin{array}{l}\text { Test Fenotípico } \\
\text { (relación IP / IP-EDTA } \geq 8)\end{array}$ & Test + & Test - & Total \\
\hline Test + & & & \\
Test - & 11 & 38 & 49 \\
Total & 11 & 10 & 10 \\
\hline
\end{tabular}

Etest se considera positivo para MBLs cuando la relación entre CIM de imipenem/imipenem -EDTA (IP/IPEDTA) es mayor o igual a 8.

Tabla 2. CIM de imipenem y relación imipenem/imipenem-EDTA, según resultado de RPC para el gen bla

\begin{tabular}{|lcc|}
\hline & bla $_{\mathrm{VIM}}+$ & bla $_{\mathrm{VIM}}-$ \\
\hline $\mathrm{n}$ & 11 & 48 \\
$\begin{array}{l}\text { CIM Imipenem } \\
\text { Mediana }\end{array}$ & 16 & 16 \\
$\begin{array}{l}\text { Relación IP/IP-EDTA } \\
\text { Mediana } \\
\text { (intervalo de confianza 95\%) }\end{array}$ & $(12,8-256)$ & $(12-16)$ \\
\hline
\end{tabular}

No se observa diferencia significativa entre la CIM de imipenem y la presencia de bla ${ }_{\mathrm{VIM}}$ Sin embargo, la relación imipenem/imipenem-EDTA (IP/IP-EDTA) fue significativamente superior en las cepas bla $_{\text {VIM }}$ positivas por RPC. * $\mathrm{p}=0,0105 \mathrm{vs} \mathrm{bla}_{\mathrm{VIM}}$ - (test Wilcoxon para muestras independientes).

electroforesis en gel de campo pulsado, como en RAPD (con partidores ERIC2) (Figuras 3 y 4).

La susceptibilidad para amikacina, ceftazidima, ciprofloxacino, y la combinación cefoperazona/ sulbactam fue menor en las cepas de Pseudomonas aeruginosa positivas para el gen $\mathrm{bla}_{\mathrm{VIM}}$, siendo la diferencia significativa sólo para cefoperazona/sulbactam En el caso de meropenem y aztreonam se observó un mayor porcentaje de cepas susceptibles en aquellas cepas portadoras de bla $_{\mathrm{VIM}}$, sin embargo, estas diferencias no fueron estadísticamente significativas (Figura 5).

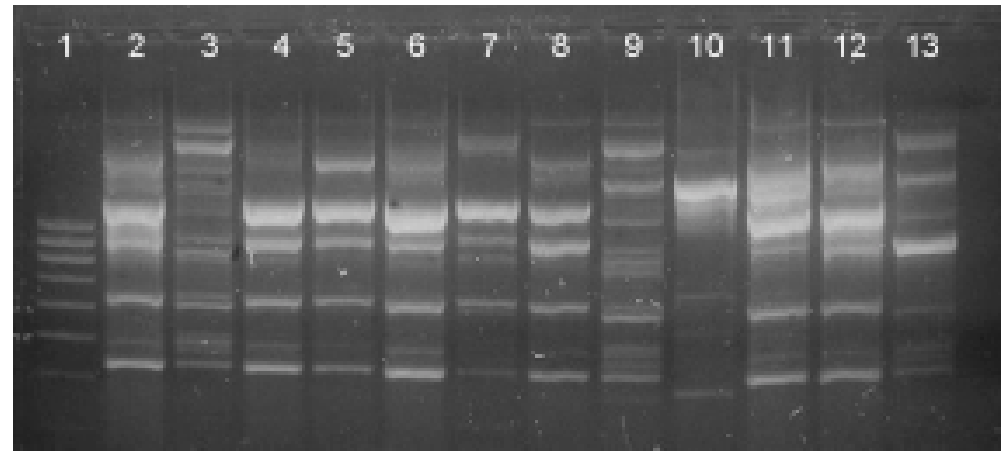

Figura 3. RAPD (partidor ERIC-2) en cepas bla ${ }_{\mathrm{VIM}}$ positivas. Carril 1 marcador de peso molecular, carriles 2-10, 12, 13: cepas de Pseudomonas aeruginosa portadoras del gen bla $_{\text {VIM. }}$. No se observaron cepas relacionadas. Carril no 6 cepa MR-4, proveniente del Hospital Militar de Santiago y carril $n^{\circ} 11$ corresponde a Pseudomonas putida OA 6820015 no incluidas en el análisis. 


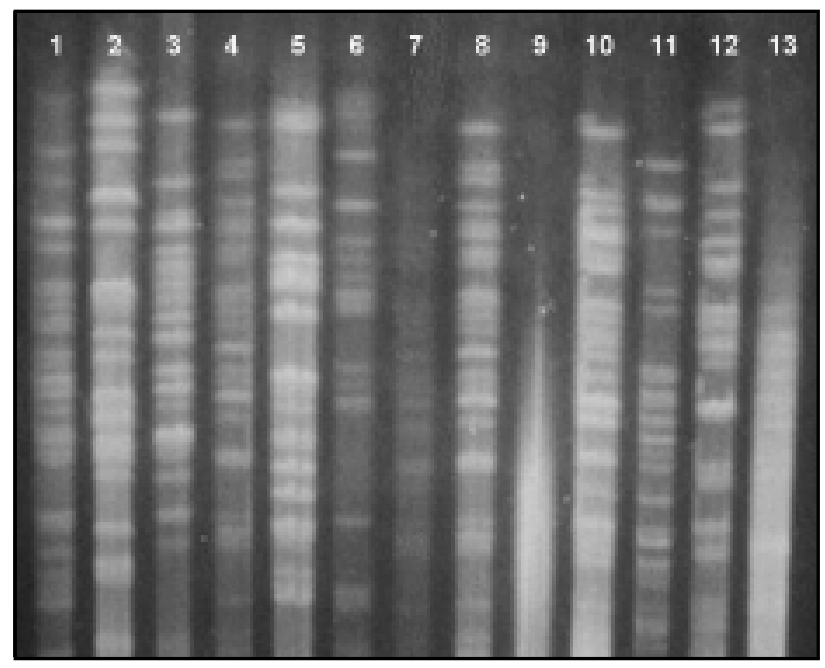

Figura 4: Electroforesis en gel de campo pulsado en cepas bla ${ }_{\mathrm{VIM}}$ positivas. Carriles 1-10,12: cepas portadoras del gen bla ${ }_{\mathrm{VIM}}$, 13 cepa ATCC. Carril 11 Pseudomonas putida OA 6820015 y carril 12 cepa MR-4, proveniente del Hospital Militar de Santiago no incluidas en el análisis. No se observan cepas relacionadas.

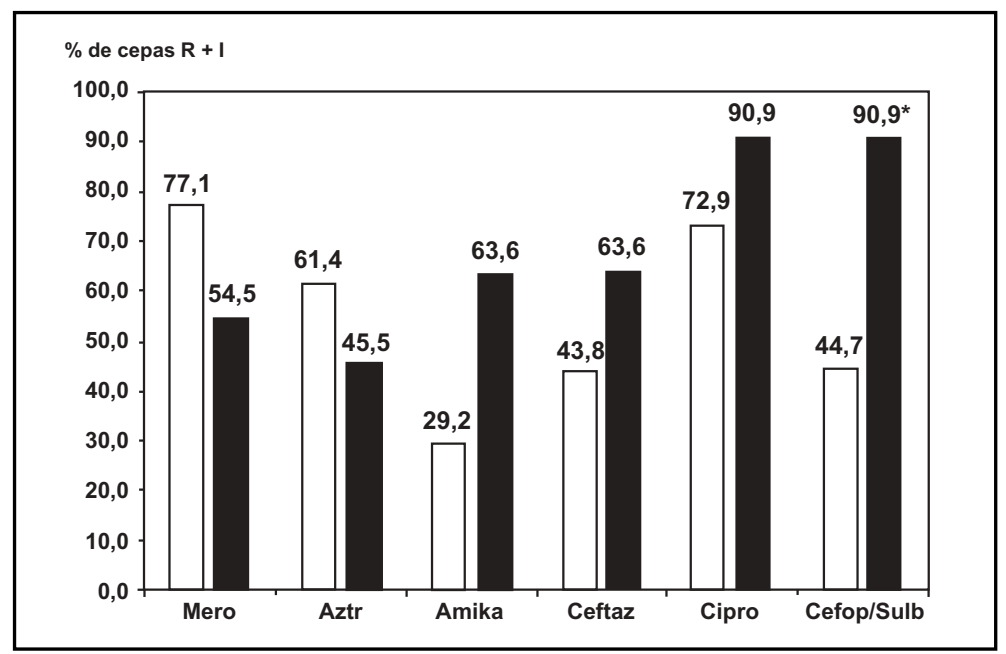

Figura 5: Pseudomonas aeruginosa resistente a imipenem: susceptibilidad disminuida a otros antibióticos en cepas bla ${ }_{\mathrm{VIM}}$ negativas y positivas. Porcentaje de cepas con susceptibilidad disminuida (resistentes (R) y con resistencia intermedia (I)) a meropenem (Mero), aztreonam (Aztr), amikacina (Amika), ceftazidima (Ceftaz), ciprofloxacino (Cipro) y cefoperazona/sulbactam (Cefop/Sulb) $\mathbf{\square}$ : cepas bla VIM $^{+}, \square$ : cepas bla VIM $^{-.}{ }^{*} \mathrm{p}$ $=0,0150$ vs bla ${ }_{\mathrm{VIM}}-$ (Test Chi-cuadrado).

Frente a colistin se analizaron cepas bla ${ }_{\mathrm{VIM}}$ positivas y negativas en conjunto, siendo todas las estudiadas susceptibles por el método de difusión ${ }^{26}$ y por Etest ${ }^{29}$.
El análisis de la multirresistencia mostró que las cepas portadoras del gen bla $\mathrm{VIM}_{\mathrm{V}}$ fueron en promedio, resistentes a un mayor número de antibióticos que las cepas no portadoras, diferencias estadísticamente significativas (Figura 6). 


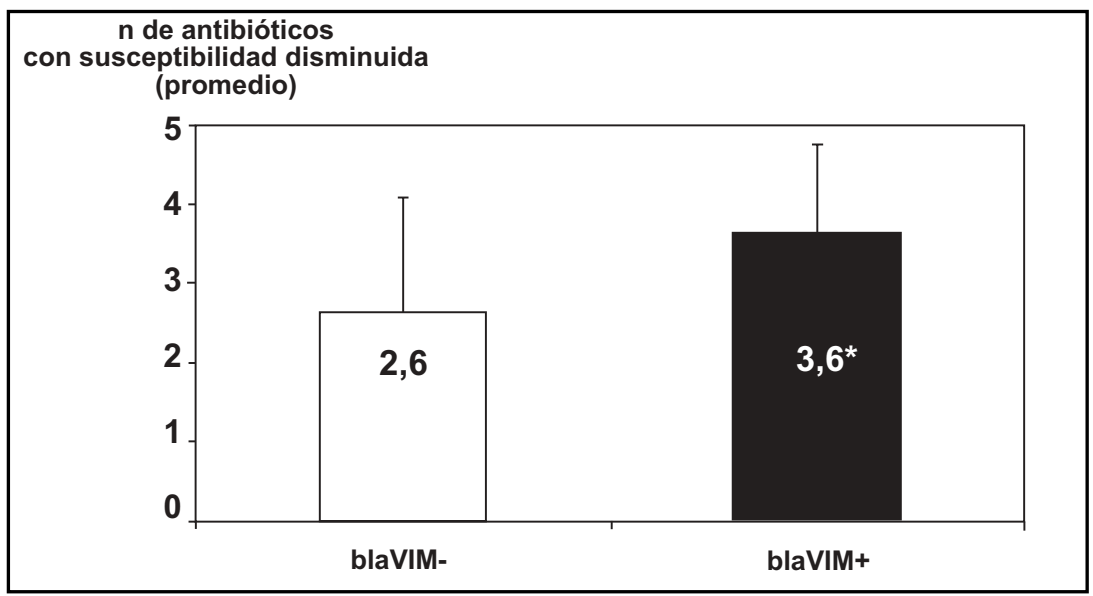

Figura 6: Multirresistencia en Pseudomonas aeruginosa bla ${ }_{\mathrm{VIM}}$ negativas y positivas: número de antibióticos con susceptibilidad disminuida (promedio). Se estudió la multirresistencia según el número de antibióticos con susceptibilidad disminuida (resistentes o con resistencia intermedia) considerando meropenem, amikacina, ceftazidima, ciprofloxacino y la combinación cefoperazona/sulbactam. Promedio y desviación estándar (barras). ${ }^{*} \mathrm{p}=0,0365$ vs bla $\mathrm{VIM}^{-}(\mathrm{t}-\mathrm{Test})$.

\section{DisCUSIÓN}

Si bien las MBLs ya habían sido descritas en Pseudomonas aeruginosa en otros países de América Latina ${ }^{12,27,28}$ éste es el primer estudio que demuestra su presencia en Chile. En este estudio, el porcentaje de cepas de $\mathrm{P}$ aeruginosa resistente a imipenem alcanzó 15,3\%; de éstas, 18,6\% es mediado por MBLs, siendo todas del tipo VIM, Si bien se estudió solamente la presencia de MBLs tipo VIM e IMP, es poco probable que GIM y SPM sean responsables de la resistencia a imipenem en las cepas bla $_{\mathrm{VIM}}$ negativas, ya que sólo han sido descritas en forma endémica en Alemania y Brasil, respectivamente $^{12,13}$. Si se extrapola el $15,3 \%$ de resistencia a imipenem por MBL encontrado en nuestro estudio, al total de cepas de Pseudomonas aeruginosa aisladas, 3\% de $\mathrm{P}$ aeruginosa sería resistente a imipenem por este mecanismo.

La resistencia a imipenem en aquellas cepas no portadoras de VIM podría ser explicada por mutaciones de la porina Opr-D, la desrepresión del gen de la B-lactamasa AmpC, o la sobreexpresión de la bomba de eflujo MexAB ${ }^{10}$, mecanismos de resistencia no considerados en este trabajo.

En nuestro estudio, el método fenotípico (Etest MBL) fue muy sensible para detectar MBLs, pero la concordancia entre ambos métodos fue de $35,6 \%$, ya que se observó un alto porcentaje de cepas con test fenotípico positivo y RPC negativo para los genes bla ${ }_{\text {VIM }}$ e bla ${ }_{\text {IMP }}$ (38 de 48 cepas, $79,2 \%$ de falsos positivos). Generalmente, se describe al Etest MBL como un método altamente sensible y específico, con valores para Pseudomonas aeruginosa con rangos que van de $87-99 \%$ y 91-97,3\%, respectivamente ${ }^{29-32}$. Los distintos valores de sensibilidad y especificidad encontrados en este estudio podrían ser explicados por los siguientes fenómenos: se ha observado que el zinc tiene un efecto directo sobre imipenem, favoreciendo su hidrólisis, por lo que quelantes de cationes divalentes como EDTA pueden crear un ambiente favorable para el imipenem y así tener un mayor efecto sobre $\mathrm{P}$ aeruginosa disminuyendo su CIM, sin ser esta reducción de la CIM producto de la inhibición de una metalo-betalactamasa ${ }^{33}$. Además, un ambiente rico en zinc tendría un efecto negativo en la porina OprD, disminuyendo su expresión y también la entrada de carbapenémicos desde el espacio extracelular, afectando también la CIM a imipenem ${ }^{34}$. Por esto, en aquellas cepas fenotípicamente productoras de MBLs por el test de sinergia imipenem-EDTA es recomendable realizar estudios confirmatorios 
más específicos como la reacción en cadena de polimerasa.

Es conocido que las MBLs son codificadas en cassettes génicos en integrones de clase 1 (sistemas de recombinación génica en bacterias, en que se ensamblan varios genes detrás de un mismo promotor) en que la resistencia a un antibiótico depende de la posición del cassette con respecto al promotor común del integrón. Así los cassettes ubicados más cerca del promotor tendrán una mayor expresión y por lo tanto mayores niveles de resistencia ${ }^{11,35}$. Esto podría explicar el amplio rango de CIMs a imipenem encontrado en las cepas productoras de VIM, desde $8 \mathrm{a} \geq 256 \mathrm{ug} / \mathrm{ml}$, hecho previamente descrito por otros autores $^{11,36,37}$. Otra explicación a esto sería la presencia de distintos subtipos de VIM ya que VIM-1 y VIM-2 muestran distintas velocidades de hidrólisis para imipenem y meropenem ${ }^{11}$.

Aunque no se encontraron diferencias significativas, se observó un mayor porcentaje de cepas con susceptibilidad disminuida a amikacina en las cepas bla $_{\text {VIM }}$ positivas. Esto puede deberse a que el cassette génico de VIM puede ir asociado en el integrón a otro cassette portador de aminopeptidasas, generando resistencia a aminoglicósidos ${ }^{10}$.

El menor porcentaje de cepas susceptibles a cefoperazona/sulbactam mostrado por las cepas bla $_{\text {VIM }}$ positivas, concuerda con lo descrito en la literatura, ya que las MBLs no resultan inhibidas por los inhibidores de ß-lactamasas (ácido clavulánico, tazobactam o sulbactam $)^{10}$.

Los distintos patrones génicos obtenidos por RADP (ERIC 2) y PFGE encontrados en las cepas positivas para el gen bla $_{\mathrm{VIM}}$ sugieren que no existió diseminación clonal. La transferencia del

\section{REFERENCIAS}

1. Gales A, Sader H, Jones R. Respiratory tract pathogens isolated from patients hospitalized with suspected pneumonia in Latin America: frequency of occurrence and antimicrobial susceptibility profile: results from the SENTRY Antimicrobial Surveillance Program (1997-2000). Diagn Microbiol Infect Dis 2002; 44: 301-11.

2. Gales A, Sader H, Jones R. SENTRY Participants Group (Latin America). Urinary tract infection trends in Latin American hospitals: report from gen bla ${ }_{\mathrm{VIM}}$ podría efectuarse por medio de elementos móviles de recombinación génica entre cepas de Pseudomonas aeruginosa no relacionadas. Si bien la presencia del cassette portador del gen bla ${ }_{\mathrm{VIM}}$ en un integrón no confiere movilidad por sí solo, los integrones sí pueden estar insertos en elementos móviles como plasmidios o transposones $^{11}$. Se ha descrito que el gen bla ${ }_{\text {VIM }}$ puede ser transferido a $\mathrm{P}$ aeruginosa, desde otras especies del género Pseudomonas, Serratia marcescens o incluso bacterias ambientales no patógenas para el hombre ${ }^{10,37}$. En 2003 se describió por primera vez en Chile un aislado clínico de Pseudomonas fluorescens portadora de VIM-2 ${ }^{14}$, desde entonces, el gen pudo ser transferido directamente a Pseudomonas aeruginosa o a través de otras bacterias ambientales no necesariamente patógenas para el hombre.

Este trabajo describe por primera vez en Chile la presencia de MBLs del tipo VIM en cepas de Pseudomonas aeruginosa aisladas en un centro hospitalario universitario, correspondiendo su frecuencia a $18,6 \%$ de las cepas resistentes a imipenem.

Es importante destacar que 2 cepas positivas para el gen bla ${ }_{\mathrm{VIM}}$ fueron aisladas de pacientes ambulatorios, sin historia de hospitalizaciones previas en nuestro hospital en 5 años. Es de suponer que estos pacientes adquirieron la bacteria portadora del gen en otro centro hospitalario.

La presencia de estas enzimas en otros centros hospitalarios de nuestro país, la determinación de los subtipos, así como la demostración de su incorporación en cassettes génicos dentro de integrones debiera ser objetivo de futuras investigaciones.

the SENTRY antimicrobial surveillance program (1997-2000). Diagn Microbiol Infect Dis 2002; 44: 289-99.

3. Sader H, Jones R, Silva J. SENTRY Participants Group (Latin America). Skin and soft tissue infections in Latin American medical centers: four-year assessment of the pathogen frequency and antimicrobial susceptibility patterns. Diagn Microbiol Infect Dis 2002; 44: 281-8.

4. Sader H, Jones R, Andrade-Baiocchi S, Biedenbach D. SENTRY Participants Group (Latin America). Four-year evaluation of frequency of occurrence 
and antimicrobial susceptibility patterns of bacteria from bloodstream infections in Latin American medical centers. Diagn Microbiol Infect Dis 2002; 44: 273-80.

5. Paramythiotou E, Lucet J, Timsit J, Vanjak D, Paugam-Burtz C, Trouillet J et al. Acquisition of multidrug-resistant Pseudomonas aeruginosa in patients in intensive care units: role of antibiotics with antipseudomonal activity. Clin Infect Dis 2004; 38: 670-7.

6. Trucco O, Prado V, Durán C, Grupo pronares. Red de vigilancia de resistencia antimicrobiana PRONARES: Informe primer semestre 2001. Rev Chil Infect 2002; 19 Suppl 2: S140-8.

7. Zambrano A, Herrera N. Susceptibilidad antimicrobiana de cepas de Pseudomonas aeruginosa aisladas en el laboratorio del Hospital Regional Dr. Leonardo Guzmán de Antofagasta, Chile. Rev Chil Infect 2004; 21: 117-24.

8. García P, Castillo C, Villagra N, Mella G, Salinas AM, Canales M, et al. Presencia de Metalo ß Lactamasas en Pseudomonas aeruginosa resistente a carbapenémicos. CO-44, página 63 Libro de Resúmenes del XXI Congreso Chileno de Infectología. Octubre de 2004. Viña del Mar, Chile.

9. Окамото K, Gotoh N, Nishino T. Pseudomonas aeruginosa reveals high intrinsic resistance to penem antibiotics: penem resistance mechanisms and their interplay. Antimicrob Agents Chemother 2001; 45: 1964-71.

10. Livermore D. Multiple mechanisms of antimicrobial resistance in Pseudomonas aeruginosa: our worst nightmare? Clin Infect Dis 2002; 34: 634-40.

11. Walsh T, Toleman M, Poirel L, Nordmann P. Metallo-B-Lactamases: the Quiet before the Storm? Clin Microbiol Rev 2005; 18: 306-25.

12. Toleman M, Simm A, Murphy T, Gales A, Biedenbach D, JONEs R ET AL. Molecular characterization of SPM-1, a novel metallo-beta-lactamase isolated in Latin America: report from the SENTRY antimicrobial surveillance programme. J Antimicrob Chemother 2002; 50: 673-9.

13. Castanheira M, Toleman M, Jones R, Schmidt F, Walsh T. Molecular characterization of a betalactamase gene, blaGIM-1, encoding a new subclass of metallo-beta-lactamase. Antimicrob Agents Chemother 2004; 48: 4654-61.

14. Mendes R, García P, Guzmán M, Toleman M, Walsh T, Jones R. First Isolation of blaVIM-2 in Latin America: Report from the SENTRY Antimicrobial
Surveillance Program. Antimicrob Agents Chemother 2004; 48: 1433-4.

15. Linden P, Kusne S, Coley K, Fontes P, Kramer D, PATERSON D. Use of parenteral colistin for the treatment of serious infection due to antimicrobial-resistant Pseudomonas aeruginosa. Clin Infect Dis 2003; 37: e154-60. Epub 2003 Oct 29.

16. Fritsche T, Sader H, Toleman M, Walsh T, Jones R. Emerging Metallo-B-Lactamase-Mediated Resistance, A Summary Report from the Worldwide SENTRY Antimicrobial Surveillance Program. Clin Infect Dis 2005; 41 Suppl 4: S276-8.

17. Yatsuyanagi J, Saito S, Harata S, Suzuki N, Ito Y, Онта K, Еломото K. Class 1 Integron Containing Metallo-B-Lactamase Gene bla $\mathrm{VIM}_{2}$ in Pseudomonas aeruginosa Clinical Strains Isolated in Japan. Antimicrob Agents Chemother 2004; 48: 626-8.

18. Ito H, Arakawa Y, Ohsuka S, Wacharotayankun R, Kато N, Онта M. Plasmid-mediated dissemination of the metallo-beta-lactamase gene blaIMP among clinically isolated strains of Serratia marcescens. Antimicrob Agents Chemother 1995; 39: 824-9.

19. Maslow J, Slutsky A, Arbeit R. Application of pulsed-field gel electrophoresis to molecular epidemiology. In: Persing DH, Smith TF, Tenover FC, White TJ. Diagnostic molecular microbiology: principles and applications. Washington, DC: American Society for Microbiology 1993: 563-72.

20. Tenover F, Arbeit R, Goering R, Mickelsen P, Murray B, Persing D, Swaminathan B. Interpreting chromosomal DNA restriction patterns produced by pulsed-field gel electrophoresis: criteria for bacterial strain typing. J Clin Microbiol 1995; 33: 2233-9.

21. Spejer H, Savelkoul P, Bonten M, Stobberinngh E, TJHiE J. Application of Different Genotyping Methods for Pseudomonas aeruginosa in a Setting of Endemicity in an Intensive Care Unit. J Clin Microbiol 1999; 37: 3654-61.

22. National Committee for Clinical Laboratory Standards. 2003. Methods for dilution antimicrobial susceptibility de test for bacteria that grow aerobically; Approved Standard-Sixth Edition. M7A6, Vol 23 (2): 12.

23. Clinical and Laboratory Standards Institute. 2005. Performance Standards for antimicrobial Susceptibility testing; Fifteen $^{\text {th }}$ Informational Supplement. Approved Standards M100-S15, Vol 25 (1).

24. Jones R, Barry A, Packer R, Gregory W, ThornsbeRRY C. In vitro antimicrobial spectrum, occurrence 
of synergy, and recommendations for dilution susceptibility testing concentrations of the cefoperazone-sulbactam combination. J Clin Microbiol 1987; 25: 1725-9.

25. Paterson D. The Epidemiological Profile of Infections with Multidrug-Resistant Pseudomonas aeruginosa and Acinetobacter Species. Clin Infect Dis 2006; 43 Suppl 2: S43-48.

26. Falagas M, Kasiakou S. Colistin: The Revival of Polymyxins for the Management of MultidrugResistant Gram-Negative Bacterial Infections. Clin Infect Dis 2005; 40: 1333-41.

27. Pasteran F, Faccone D, Petroni A, Rapoport M, Galas M, VÁzQuez M et al. Novel variant (bla(VIM-11)) of the metallo-\{beta\}-lactamase bla(VIM) family in a GES-1 extended-spectrum-B-lactamase-producing Pseudomonas aeruginosa clinical isolate in Argentina. Antimicrob Agents Chemother 2005; 49: 474-5.

28. Villegas M, lolans K, Olivera M, Suárez J, Correa A, Queenan A et al. First Detection of Metallo-BLactamase VIM-2 in Pseudomonas aeruginosa Isolates from Colombia. Antimicrob Agents Chemother 2004; 48: 4654-61.

29. Lee K, Yong D, Yum J, LIM Y, Bolmström A, Qwärnstrom A ET AL. Evaluation of Etest MBL for Detection of bla $\mathrm{IMP}-1_{1}$ and bla $\mathrm{VIM}-2_{\text {Allele-Positive }}$ Clinical Isolates of Pseudomonas spp. and Acinetobacter spp. J Clin Microbiol 2005; 43: 942-4.

30. Walsh T, Bolmström A, Qwärnström A, Gales A. Evaluation of a New Etest for Detecting Metallo-
ß-Lactamases in Routine Clinical Testing. J Clin Microbiol 2002; 40: 2755-9.

31. Pitout, J, Gregson D, Poirel L, McClure J, Le P, Church D. Detection Pseudomonas aeruginosa Producing Metallo-ß-Lactamases in a Large Centralizad Laboratory. J Clin Microbiol 2005: 43; 3129-35.

32. Yan J, Wu J, Tsai S, Chuang C. Comparison of the double-disk, combined disk, and Etest methods for detecting metallo-ß-lactamases in gram-negative bacilli. Diagn Microbiol Infect Dis 2004: 49; 5-11.

33. BaXter I, Lambert P. The effect of zinc on imipenem. Antimicrob Agents Chemother 1997; 39: 838-9.

34. Conejo MC, García I, Martínez-Martínez L, Picabea L, Pascual A. Zinc Eluted from Siliconized Latex Urinary Catheters Decreases OprD Expression, Causing Carbapenem Resistance in Pseudomonas aeruginosa. Antimicrob Agents Chemother 2003: 47; 2313-5.

35. Collis C, Hall R. Expression of antibiotic resistance genes in the integrated cassettes of integrons. Antimicrob Agents Chemother 1995; 39: 155-62.

36. Docquier J, Lamotte-Brasseur J, Galleni M, Amicosante G, Frere J, Rossolini G. On functional and structural heterogeneity of VIM-type metallobeta-lactamases. J Antimicrob Chemother 2003; 51: 257-66.

37. Nordmann P, Poirel L. Emerging carbapenemases in Gram-negative aerobes. Clin Microbiol Infect 2002; 8: 321-31. 